\title{
Operational modal analysis and Bayesian model updating of a standing seam metal roofing system
}

\author{
Jun Hu and Paul Heung-Fai Lam* \\ Department of Architecture and Civil Engineering, \\ City University of Hong Kong, Hong Kong \\ Email: juhoo.19880203.whu@gmail.com \\ Email: paullam@cityu.edu.hk \\ *Corresponding author
}

\section{Kwok-Fai Chung}

Chinese National Engineering Research Centre for

Steel Construction (Hong Kong Branch),

The Hong Kong Polytechnic University, Hong Kong

Email: kwok-fai.chung@polyu.edu.hk

\begin{abstract}
Clip detachment is found to be a common failure mode for metal roofs. It leads to the stiffness reduction at some supports of the metal roof and alter its dynamic characteristics. Therefore, it is possible to detect the damage of clips by monitoring the modal properties. To assess the dynamic properties of metal roofs, part of a full-scale standing seam metal roof was constructed on a supporting steel frame in the laboratory. Modal parameters were identified from the measured acceleration responses using frequency domain decomposition method (FDD). The finite element model of the system was developed, and the clip stiffness was estimated by Markov chain Monte Carlo (MCMC)-based Bayesian model updating method. Both the modal identification and model updating results are reported in detail. The results presented in this paper are valuable and important for the development of a structural health monitoring system for metal roofing system.
\end{abstract}

Keywords: operational modal analysis; standing seam metal roofing system; Bayesian model updating; Markov chain Monte Carlo simulation.

Reference to this paper should be made as follows: Hu, J., Lam, P.H-F. and Chung, K-F. (2019) 'Operational modal analysis and Bayesian model updating of a standing seam metal roofing system', Int. J. Lifecycle Performance Engineering, Vol. 3, No. 1, pp.4-19.

Biographical notes: Jun $\mathrm{Hu}$ is currently a Research Associate in in the Department of Architecture and Civil Engineering at the City University of Hong Kong. His research interests are full-scale ambient test, operational modal analysis, Bayesian structural model updating and vibration motoring.

Paul Heung-Fai Lam is currently an Associate Professor in the Department of Architecture and Civil Engineering at the City University of Hong Kong, President of the Hong Kong Society of Theoretical and Applied Mechanics (HKSTAM) and the Chairman (Education and Training) of the Hong Kong Construction Metal Structures Association (HKCMSA). His research interests 


\begin{abstract}
are structural dynamics, vibration measurement, modal identification, structural model updating and damage detection, Bayesian system identification, design and analysis of vertical axis wind turbine.

Kwok-Fai Chung is currently the Associate Head of Department of Civil and Environmental Engineering at the Hong Kong Polytechnic University, and Director of Chinese National Engineering Research Centre for Steel Construction (Hong Kong Branch) endorsed by the State Ministry of Science and Technology in China. He is also the Founding President of the Hong Kong Constructional Metal Structures Association. He is a renowned academic, researcher and structural engineer with established expertise in steel construction. His research interests include limit state analysis and performance-based design of structural steel systems, structural fire engineering and fire protection in buildings and tunnels, and design codification.
\end{abstract}

This paper is a revised and expanded version of a paper entitled 'Vibration test and finite element modeling of a steel frame supported standing seam metal roof panel' presented at ICSAS 2016, Hong Kong, China, 7-9 December.

\title{
1 Introduction
}

A standing seam metal roofing system can be characterised as an assembly of metal panels (usually made of steel or aluminium) adjacently connected to each other using a roll-forming machine (see Figure 1 as an example). The edges of the adjacent metal panels are rolled along to form a double lock seam, called 'standing seam', providing an excellent water tightness lock seam that stands above the run-off water line. A standing seam metal roofing system is among the most cost-efficiency and durable of existing roofing systems. It can be designed with various types of aesthetical roof profiles and the construction process is fast and easy. Therefore, the standing seam metal roofing system has been widely adopted in public facilities, such as airport terminals, railway stations and exhibition halls. The metal panels are supported by roof purlins through clip fasteners along the seam lock, forming a stable seam-clip-purlin connection. Current researches mainly focus on the performance of metal roofs under wind load by undertaking static property evaluation of various structural components under design loads. In Serrette and Pekoz (1995a), the analytical estimation for the elastic distortional buckling stress of metal panels based on elastic foundation model is presented. The proposed formulation was verified by the comparison between the analytical results and the results from elastic finite-strip buckling experiment. Based on the proposed formulation, Serrette and Pekoz (1995b) proposed two design methods for the interaction between local and distortional buckling of metal panels. The proposed new design methods were proved more accurate in the approximation of the maximum resistance of standing seam metal panels. Later, Sinno et al. (2001) discovered that the standing seam metal roofing system is susceptible to wind pressure and the uplifting effect of wind force is one of the main causes of clip damage of the metal roofs. To simulate this effect, a magnetic uplift load test was carried out. Farquhar et al. (2005) carried out a series of tests on a scaled standing seam metal roof, which was designed to fail at clip, to quantify the relationship between uniform 
uplift pressure using pressure chamber and the dynamic pressures of real wind loading using wind tunnel test. Based on the test results, an effective external pressure coefficient was proposed. To understand the uplift behaviour of standing seam metal roofs, Damatty et al. (2003) developed a three-dimensional finite element model of a standing seam roof, in which the seam and clip were modelled by equivalent springs. In the modelling of standing seam metal roof, the most challenging part is the modelling of connections between adjacent metal panels (i.e., the seam joint) and the connections among the metal panels and the clips (i.e., the seam-clip-purlin joint). To quantify the stiffness values of the equivalent springs, a series of static experiments were conducted.

Like all types of roof systems, standing seam metal roofing system is susceptible to wind load and clip detachment is the most common failure mode. Visual inspection for potentially damaged clips is extremely difficult because clips are often hidden by the panels and purlins. Water leakage accompanied by a wind storm through a failed roofing system can lead to severe damage to interior essential facilities and occupants. Moreover, undetected damage may accumulate and increase the chance of unexpected catastrophic collapse. One alternative solution for the detection of clip damage is to conduct a continuously vibration monitoring to obtain the vibration responses and identify the clip stiffness of the system by structural model updating using the identified modal parameters. Structural model updating utilising measured vibration responses has been applied in numerous types of structures (Yuen and Katafygiotis, 2003; Feng and Feng, 2017), while very few researches have been carried out on metal roofing system. This paper presents a step by step work for the ambient vibration test, modal identification and model updating of a full-scale standing seam metal roof panel. The test panel was constructed in the heavy structures testing laboratory of the City University of Hong Kong (CityU). The ambient vibration condition was simulated using an electricity fan and the modal parameters were identified following the frequency domain decomposition (FDD) method. The Markov chain Monte Carlo (MCMC)-based Bayesian model updating method was applied in the model updating to quantify the stiffness of clip. A finite element model of the test panel was built and updated using the identified modal parameters. Finally, the measured and model-predicted results were compared and discussed. The research findings are helpful in the understanding of the vibration characteristics and the development of an accurate finite element model for capturing the dynamic characteristics of this kind of roofing system in the future.

\section{Indoor panel test and experimental results}

\subsection{Indoor panel test}

Figure 1(a) (Hu et al., 2016) shows the indoor test panel constructed in the laboratory. The full-scale test panel is comprised of three full-size metal strips at the middle together with two half-size strips at the two edges. The test panel is connected to the three purlins using 12 clips on top of a steel frame. Figure 2 shows the clip employed to connect the metal roof to the purlin of the steel frame. The dimension of the indoor test panel is $1.84 \mathrm{~m}$ width by $2.7 \mathrm{~m}$ long, as shown in Figure 1(b), in which the red dots and blue crosses show the locations of sensors on the test panel and the locations of clips, respectively. In total, 18 measurement locations were planned. The vibration time history 
data were measured by 18 uniaxial accelerometers with a sampling frequency of $2,048 \mathrm{~Hz}$ in a single setup. To simulate the ambient vibration condition, an electricity fan was used to generate wind load on the test panel [see Figure 1(a)]. Figure 3 shows the measured vibration at the first channel (the measured signals at other channels are similar). It is clear from Figure 3 that the largest amplitude of vibration is about $0.1 \mathrm{~g}$.

Figure 1 The metal roof test panel, (a) the physical panel supported by steel purlins (b) the dimensions (in m), sensor locations (red dots) and clip locations (blue crosses) (see online version for colours)

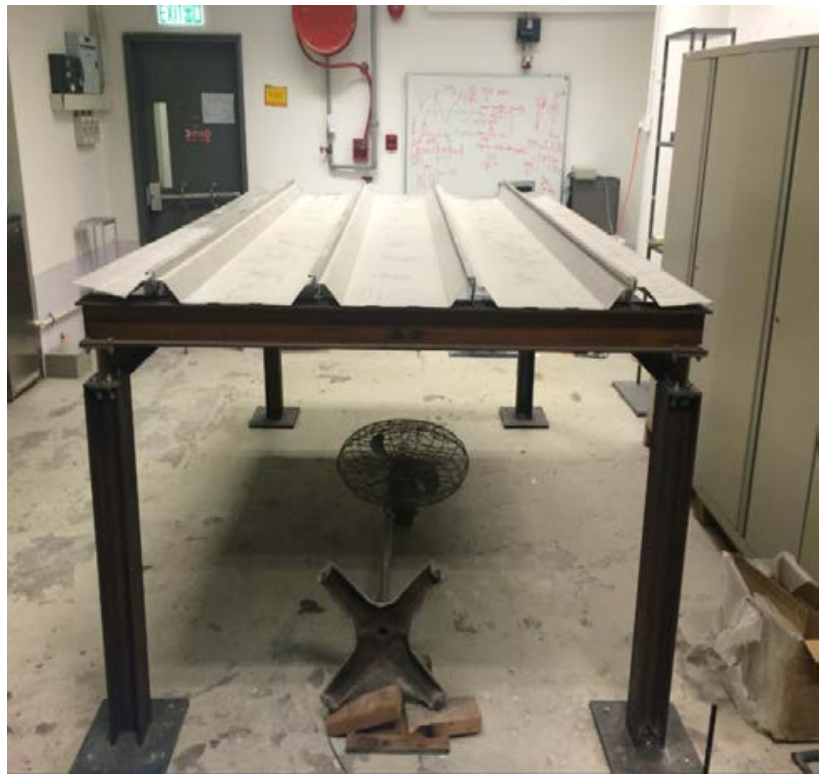

(a)



(b)

Note: (a) The indoor test panel and (b) the measurement plan. Source: Hu et al. (2016)

Figure 2 The clip employed in connecting the metal roof onto the purlin (see online version for colours)




Figure 3 Time-history data from the first measurement channel (see online version for colours)

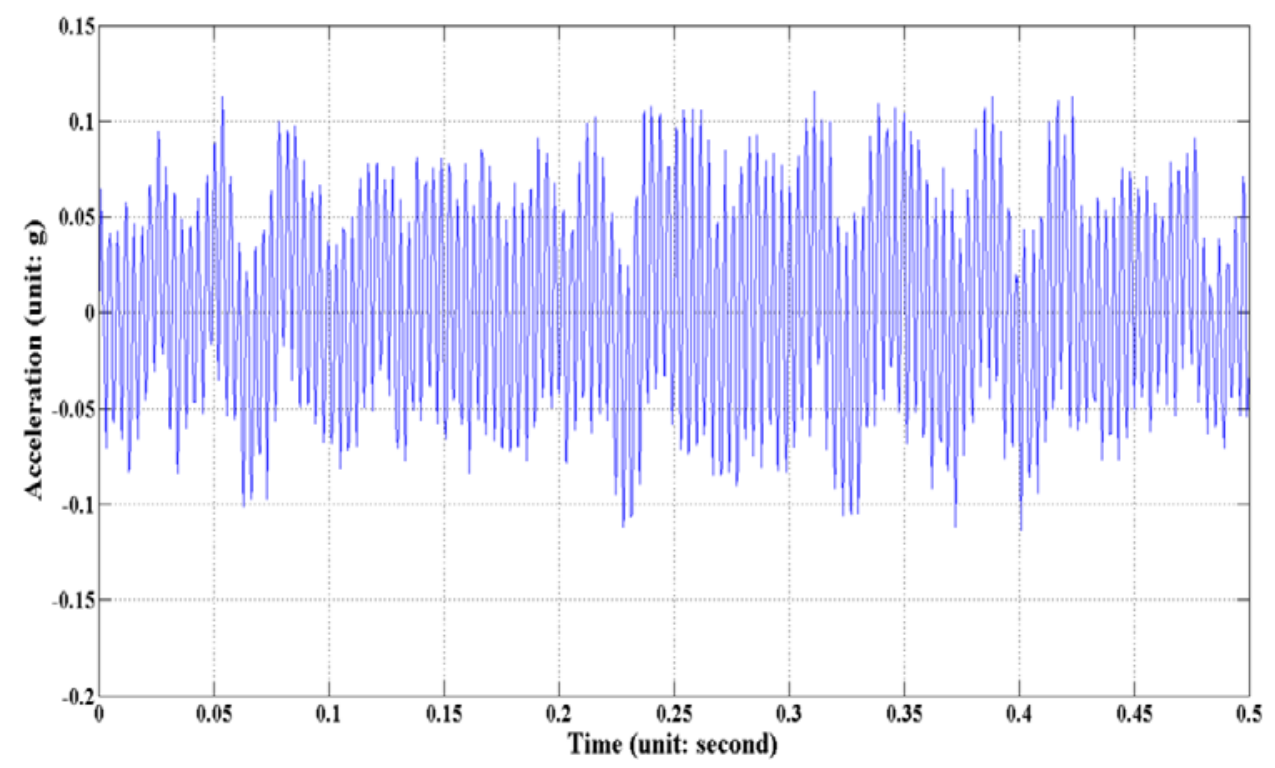

\subsection{Modal identification using FDD}

To extract the modal parameters from the measured data, FDD is adopted in this paper. To be self-contained, the basic theory of FDD is briefly reviewed in this section. Interested readers should refer to the original development (Brincker et al., 2001). At frequency $\omega$, the power spectrum density (PSD) matrix is written as

$$
\mathbf{G}_{y}(\mathrm{i} \omega)=\overline{\mathbf{H}}(\mathrm{i} \omega) \mathbf{G}_{x}(\mathrm{i} \omega) \mathbf{H}^{T}(\mathrm{i} \omega)
$$

where $\mathbf{G}_{y}(\mathrm{i} \omega)$ is the $n$ by $n$ PSD matrix of the measured output responses; $n$ is the number of measured degrees of freedom (DOFs), $i^{2}=-1, \mathbf{G}_{x}(\mathrm{i} \omega)$ is the $m$ by $m$ PSD matrix of the inputs, $m$ is the number of inputs, $\mathbf{H}(\mathrm{i} \omega)$ is the $n$ by $m$ frequency response function (FRF) matrix, the overbar denotes the complex conjugate and the superscript $T$ denotes the transpose. The FRF matrix is further expressed in the partial fraction form:

$$
\mathbf{H}(\mathrm{i} \omega)=\sum_{j=1}^{N_{m}} \frac{\mathbf{R}_{j}}{\mathrm{i} \omega-\lambda_{j}}+\frac{\overline{\mathbf{R}}_{j}}{\mathrm{i} \omega-\bar{\lambda}_{j}}
$$

where $N_{m}$ is the number of modes, $\lambda_{j}$ is the $j^{\text {th }}$ pole and $\mathbf{R}_{j}$ is the residue given by

$$
\mathbf{R}_{j}=\boldsymbol{\varphi}_{j} \boldsymbol{\beta}_{j}^{T}
$$

where $\boldsymbol{\varphi}_{j}$ is the mode shape of the $j^{\text {th }}$ mode and $\boldsymbol{\beta}_{j}$ is the modal participation vector of the $j^{\text {th }}$ mode. If the input is white noise, substituting equation (2) into equation (1) gives the output PSD matrix in the following form 


$$
\mathbf{G}_{y}(\mathrm{i} \omega)=\sum_{j=1}^{N_{m}} \frac{\mathbf{K}_{j}}{\mathrm{i} \omega-\lambda_{j}}+\frac{\overline{\mathbf{K}}_{j}}{\mathrm{i} \omega-\bar{\lambda}_{j}}+\frac{\mathbf{R}_{j}}{\mathrm{i} \omega-\lambda_{j}}+\frac{\overline{\mathbf{R}}_{j}}{\mathrm{i} \omega-\bar{\lambda}_{j}}
$$

where $\mathbf{K}_{j}$ is the $j^{\text {th }}$ residue matrix of the output PSD matrix. When the damping is small, it can be shown that the residue matrix is proportional to the mode shape:

$$
\mathbf{K}_{j} \propto g_{j} \boldsymbol{\varphi}_{j} \boldsymbol{\varphi}_{j}^{T}
$$

where $g_{k}$ is a constant. The output PSD matrix can then be expressed explicitly as

$$
\mathbf{G}_{y}(\mathrm{i} \omega) \approx \sum_{j=1}^{N_{m}} \frac{g_{j} \boldsymbol{\varphi}_{j} \boldsymbol{\varphi}_{j}^{T}}{\mathrm{i} \omega-\lambda_{j}}+\frac{\bar{g}_{j} \overline{\boldsymbol{\varphi}}_{j} \overline{\boldsymbol{\varphi}}_{j}^{T}}{\mathrm{i} \omega-\bar{\lambda}_{j}} .
$$

To identify modal parameters using FDD, the first step is to conduct singular value decomposition (SVD) for output PSD matrices at every frequency and construct the singular value spectra. The natural frequencies can be identified at the peaks of the curve corresponding to the largest singular values while the mode shapes can be obtained as the singular vectors of the output PSD matrices at the natural frequencies.

\subsection{Experimental results}

Figure 4 shows the first singular value spectrum calculated from the measured time-domain data from all 18 channels and only vertical vibration was considered in the modal identification analysis. From the singular value spectrum, four vertical modes were clearly observed.

Figure 4 First singular value spectrum (see online version for colours)

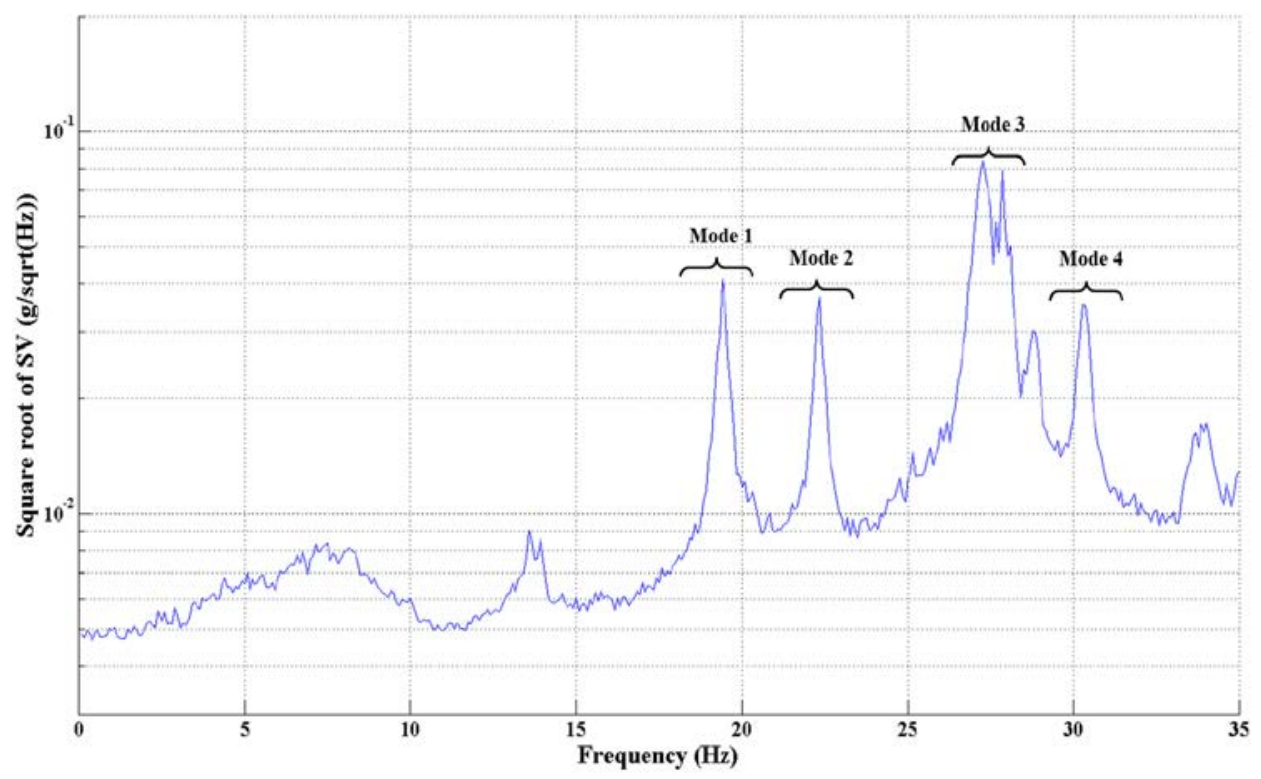


Figure 5 The identified modal parameters, (a) Mode 1: $19.42 \mathrm{~Hz}, 0.67 \%$ (b) Mode 2: $22.30 \mathrm{~Hz}$, $0.52 \%$ (c) Mode 3: $27.25 \mathrm{~Hz}, 0.57 \%$ (d) Mode 4: $30.30 \mathrm{~Hz}, 0.37 \%$ (see online version for colours)



$\mathbf{x}$-axis (m)

(a)

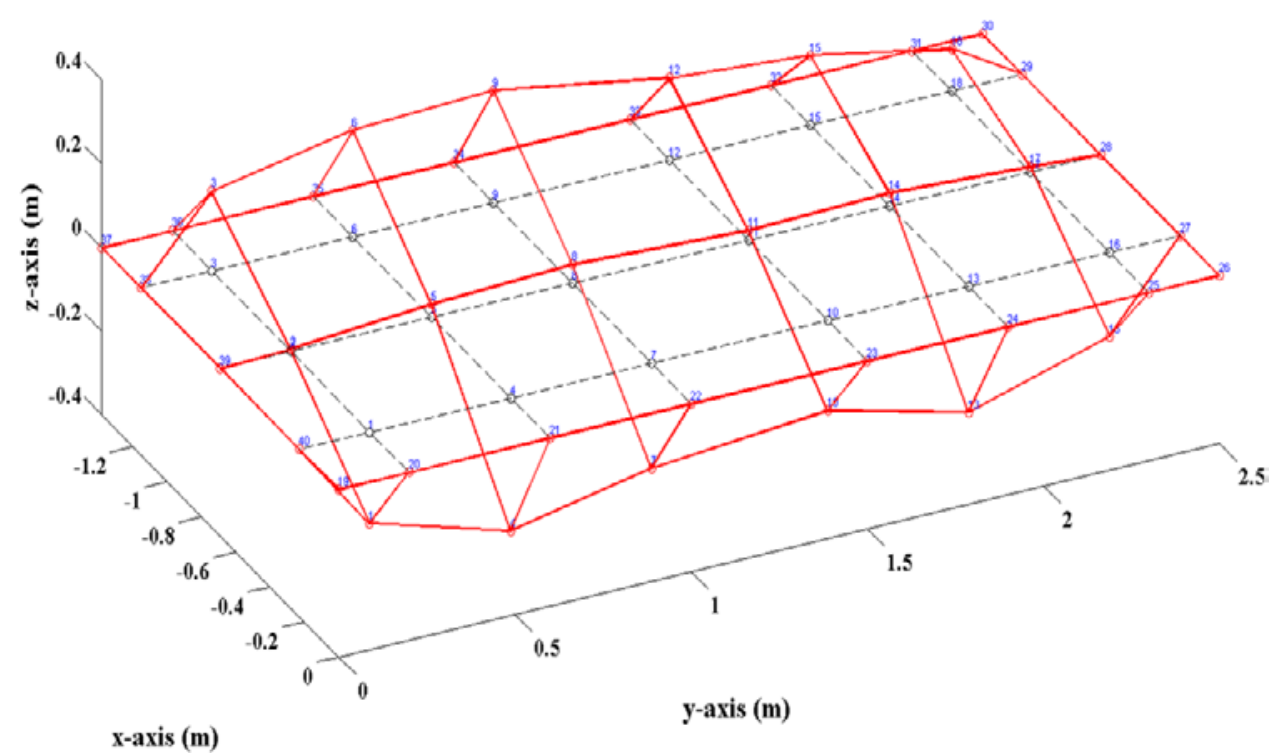

(b) 
Figure 5 The identified modal parameters, (a) Mode 1: $19.42 \mathrm{~Hz}, 0.67 \%$ (b) Mode 2: $22.30 \mathrm{~Hz}$, $0.52 \%$ (c) Mode 3: $27.25 \mathrm{~Hz}, 0.57 \%$ (d) Mode $4: 30.30 \mathrm{~Hz}, 0.37 \%$ (continued) (see online version for colours)



(c)



$\mathbf{x}$-axis (m) 
Figure 5 shows natural frequency, modal damping and mode shape of the four vertical vibration modes, which were identified from the ambient vibration data. Figure 5(a) is the first identified mode (Mode 1) with a natural frequency of $19.42 \mathrm{~Hz}$ and a damping ratio of $0.67 \%$. It is clear that when the measurement points on the centre metal strip move downward, the measurement points on the two side strips move upward. Figure 5(b) shows the second identified mode (Mode 2) with a natural frequency of 22.30 $\mathrm{Hz}$ and a damping ratio of $0.52 \%$. The vibration at the measurement locations on the centre strip is very small. However, the vibrations at the two side strips are obvious and they are in opposite directions. This mode is like a rigid body rotational mode of the panels about the centre line. Figure 5(c) depicts the third vertical modes (Mode 3) with a natural frequency of $27.25 \mathrm{~Hz}$ and a damping ratio of $0.57 \%$. The vibration of the test panel can be divided by the central axis along y-axis into two parts and they are antisymmetric. Figure 5(d) is the fourth identified mode (Mode 4) with a natural frequency of $30.30 \mathrm{~Hz}$ and a damping ratio of $0.37 \%$. Similar to Mode 3, the vibration of the entire test panel can be divided into two parts, when the front part goes upward, the back part moves downward.

\section{Finite element modelling and model updating of the metal roof test panel}

\subsection{Modelling method}

The modelling of the seam-clip-purlin connection is a challenge in the development of the numerical model of metal roofs. Since the two adjacent metal strips is not rigid connected, the relative displacements in three directions (in the global $x, y$ and $z$ directions) together with the relative rotation should be considered. To simulate those relative displacements in the FE model, the seam-clip-purlin connection between two metal strips was modelled using equivalent springs (Damatty et al., 2003) as shown in Figure 6. Since the vibration amplitude is small in this study, all springs are assumed to be linear elastic. Figure 6(a) depicts the schematic figure of the seam-clip-purlin joint model. The relative displacements in the three perpendicular directions between two adjacent metal strips are captured by the three translational springs with stiffness values $k_{x}, k_{y}$ and $k_{z}$ and the relative rotation between the two adjacent metal strips is simulated as the rotational spring with stiffness value $k_{r}$. The clip is simulated as two longitudinal springs each with a stiffness value of $k_{c} / 2$ (i.e., the total stiffness of a clip is equal to $k_{c}$ ). The top of the clip is connected to the two metal panels, whereas the bottom is connected to the purlin.

Figure 6(b) shows the schematic figure of the seam joint model far from the clip location and the modelling strategy for the relative displacement is the same as the seam-clip-purlin connection. When compared to the modelling of the seam joint and seam-clip-purlin joint, the modelling of other components of metal roofing system is relatively simple. The columns, beams and purlins of the supporting steel frame were modelled using beam element and the metal strips were modelled using shell elements in ANSYS as shown in Figure 7. 
Figure 6 Finite element modelling of (a) seam-clip-purlin and (b) seam joint (see online version for colours)

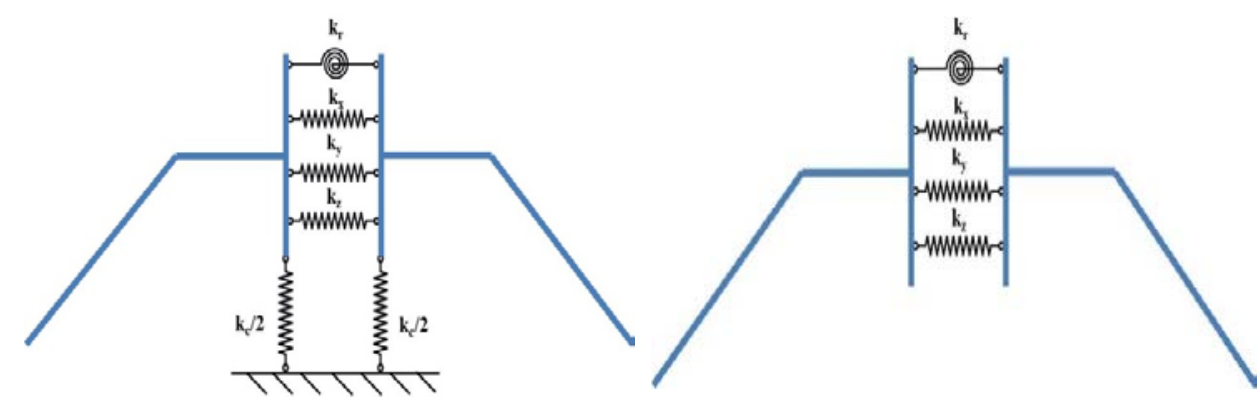

(a)

(b)

Figure 7 Finite element model of the metal roof test panel (see online version for colours)

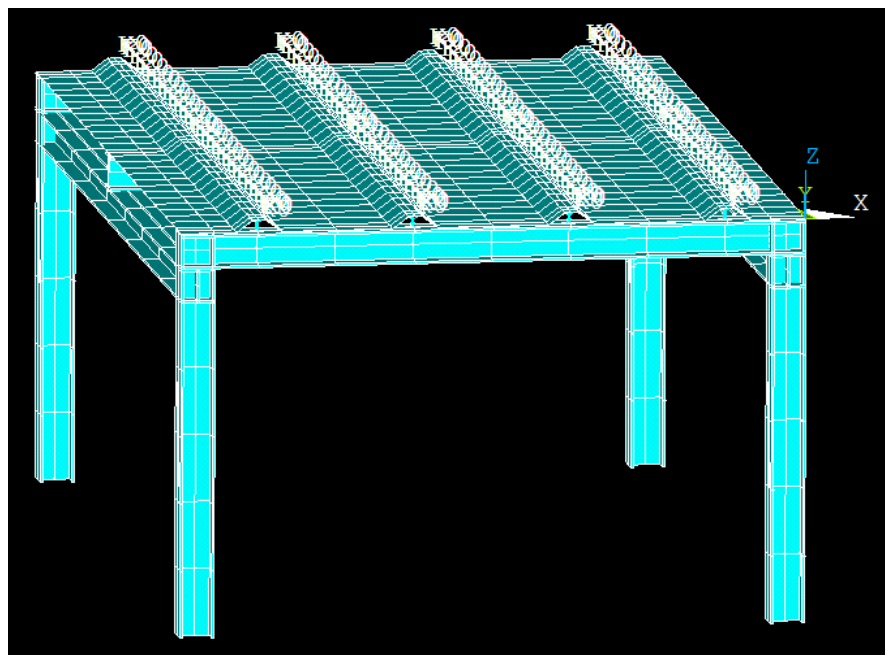

\subsection{Bayesian model updating by $M C M C$}

To be self-contained, the basic theory of the MCMC-based Bayesian model updating method is briefly introduced here. To appropriately address measurement noise and modelling error, the fractional errors of the natural frequencies and mode shapes are assumed to follow the zero-mean Gaussian distribution with variance $\kappa^{2}$. One can formulate the likelihood function as

$$
p(\boldsymbol{D} \mid \boldsymbol{\theta})=\exp \left(-\frac{J(\boldsymbol{\theta})}{2 \kappa^{2}}\right) \prod_{i=1}^{n} \frac{1}{2 \pi \kappa^{2}}
$$

where $\boldsymbol{D}$ denotes the set of identified modal parameters that is the set of measured data in the model updating process, subscript $i$ denotes the mode index, $n$ is the total number of modes to be considered in the model updating process, $\boldsymbol{\theta}$ denotes the uncertain model parameter vector and $J(\boldsymbol{\theta})$ is the measure-of-fit function given by 


$$
J(\boldsymbol{\theta})=\sum_{i=1}^{n}\left[\left(\frac{\hat{f}_{i}-f_{i}(\boldsymbol{\theta})}{\hat{f}_{i}}\right)+\left(1-\left|\hat{\mathbf{\Phi}}_{i}^{T} \boldsymbol{\Phi}_{i}(\boldsymbol{\theta})\right|^{2}\right)\right]
$$

where $\hat{f}_{i}$ denotes the measured natural frequency in $\mathrm{Hz}, f_{i}(\boldsymbol{\theta})$ is the calculated natural frequency whose dependence on uncertain model parameters $\boldsymbol{\theta}$ is emphasised and $\hat{\boldsymbol{\Phi}}_{i}$ and $\boldsymbol{\Phi}_{i}(\boldsymbol{\theta})$ denote the measured and calculated mode shapes, respectively.

The posterior PDFs of the uncertain parameters are formulated following the works presented in Lam et al. (2017a), as

$$
p(\boldsymbol{\theta} \mid \boldsymbol{D})=c \exp \left(-\frac{J(\boldsymbol{\theta})}{2 \kappa^{2}}\right)
$$

where $c$ is a normalising constant.

The bridge PDF is at the $j^{\text {th }}$ sampling level and $p_{j}$ is thus constructed according to the posterior PDF, as in (Lam et al., 2017a):

$$
p_{j}=c_{j} \exp \left(-\frac{J(\boldsymbol{\theta})}{2 \kappa_{j}^{2}}\right)
$$

where $\kappa_{j}^{2}$ controls the size of the region covered by the bridge PDF and decreases gradually from level to level. In each level, sampling is conducted using the Metropolis-Hastings (MH) algorithm (Metropolis et al., 1953; Hastings, 1970) with the proposal PDF constructed by the kernel density (Au and Beck, 1999). The important regions of the posterior PDF are gradually reached as the sampling goes on. The procedures of MCMC-based model updating are summarised below. The MCMC algorithm for Bayesian model updating can be summarised as follows:

1 Determine the algorithm parameters such as sample number $N$, sample level $l$ and changing rate $A$ of the bridge PDFs between two successive sampling levels and construct the objective function $J(\boldsymbol{\theta})$ based on the identified modal parameters.

2 In the first level, generate $N$ samples using the MH algorithm. A uniform PDF is used as the proposed PDF.

3 In a general level $j$, the sampling procedure is similar to that in the first level except that the proposed PDF in the MH algorithm is constructed by kernel density estimation based on the samples generated in the previous level $j-1$.

4 Repeat Step (3) until the samples in the final level are generated. The posterior marginal PDFs can be efficiently calculated by analytically integrating the kernel density based on the samples in the final sampling level. The posterior uncertainties can then be assessed based on the posterior marginal PDFs.

\subsection{Model updating results}

In this study, the equivalent spring between two adjacent metal strips were treated as constant and only the stiffness of clip (nominal value $k_{c} / 2=2.2 \times 10^{6} \mathrm{~N} / \mathrm{m}$ ) was considered as the uncertain model parameters. Based on the developed finite element model, the model-predicted mode shapes in the vertical direction were calculated and 
matched with the measured one. In the model updating process, the stiffness of the clip was calculated by multiplying the nominal values of $k_{c} / 2$ by the non-dimensional scaling factors $\theta_{c}$. Based on the generated MCMC samples from the Bayesian model updating, the posterior PDF of the uncertain parameters were determined. The solid line in Figure 8 shows the posterior marginal PDF of the scaling factor for the clip. The posterior marginal PDF were fitted by Gaussian distribution (the dashed line in Figure 8 to calculate the mean and COV). It is found that the MPV of the scaling factors is 1.31, which is close to unity indicating a reasonable selection of nominal value of the stiffness of clip. It is believed that a value of $5.76 \times 10^{6} \mathrm{~N} / \mathrm{m}$ can be employed as the axial stiffness, $k_{c}$, of this kind of clips for dynamic analysis. It is found that the COV of the scaling factor is $39.3 \%$, indicating a relatively large uncertainty. It is believed that modelling error is a major contributor to this result.

Figure 8 Marginal PDFs of the stiffness scaling factor for clip (see online version for colours)

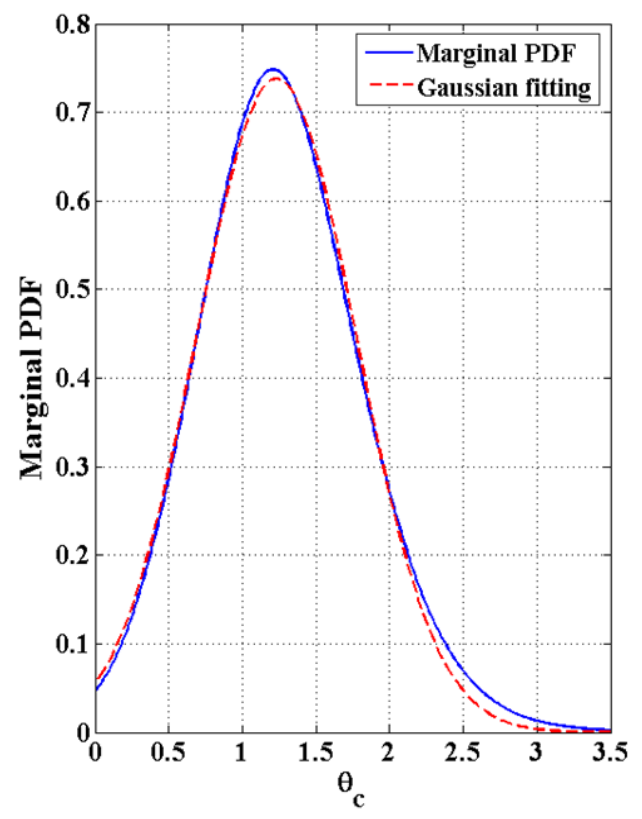

Table 1 and Table 2 show the discrepancies between the measured and model-predicted natural frequencies and mode shapes in terms of modal assurance criterion (MAC) for the four identified modes after model updating. From Table 1, the largest percentage error in natural frequency is about $5 \%$ for Mode 2 . From Table 2, it is very clear that the MAC values for all the modes are very close to $100 \%$ implying that the matchings between the measured and model-predicted mode shapes were very good. The MAC values for modes 1 and 2 are basically the same and they are a bit larger than the MAC values of modes 3 and 4 . This may be caused by the fact that the mode shapes for higher modes are usually more complicated when compared to those for lower modes. To show the accuracy of updated the finite element model, the measured mode shapes (blue solid line) are plotted together with the model-predicted ones (red dashed line) in Figure 9. The matchings between measured and model-predicted mode shapes are in general reasonable. 
Table 1 Percentage errors between the measured and model-predicted natural frequencies

\begin{tabular}{lccc}
\hline Mode & Measured & Model-predicted & Discrepancy \\
\hline Mode 1 $(\mathrm{Hz})$ & 19.42 & 19.83 & $2.11 \%$ \\
Mode 2 $(\mathrm{Hz})$ & 22.30 & 23.42 & $5.02 \%$ \\
Mode 3 $(\mathrm{Hz})$ & 27.25 & 28.14 & $3.27 \%$ \\
Mode 4 $(\mathrm{Hz})$ & 30.30 & 31.05 & $2.48 \%$ \\
\hline
\end{tabular}

Figure 9 Model-predicted mode shape for (a) Mode 1, (b) Mode 2, (c) Mode 3 and (d) Mode 4 (see online version for colours)



(a)

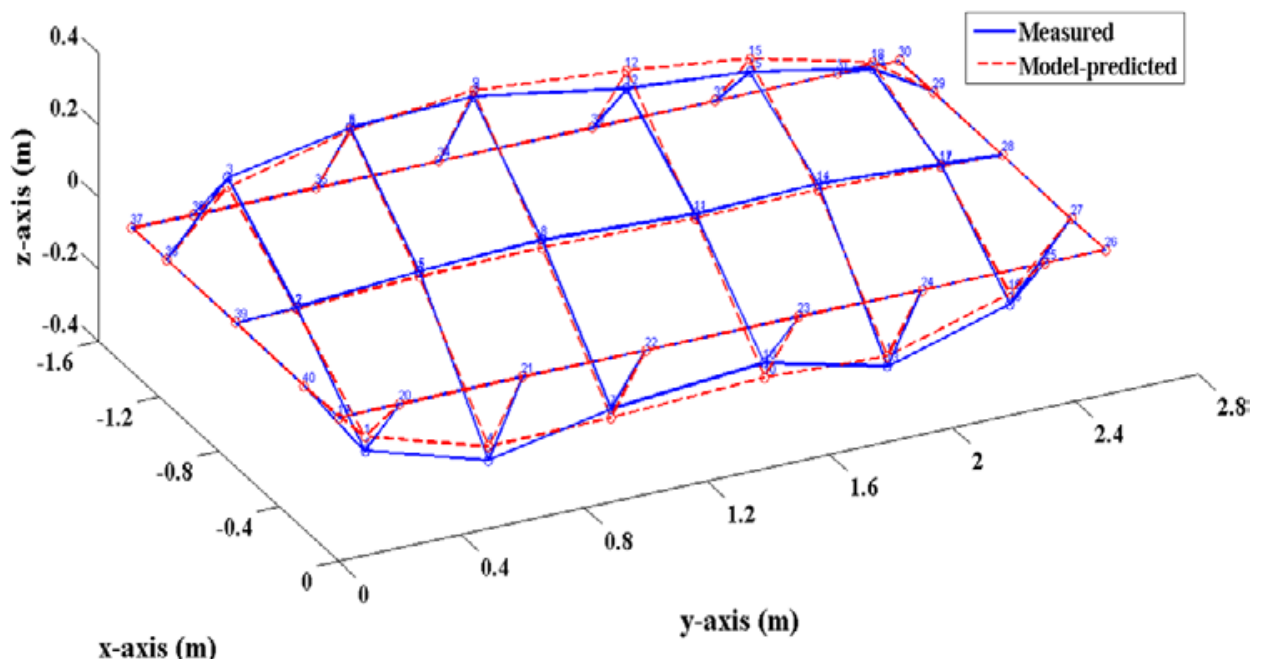

(b) 
Figure 9 Model-predicted mode shape for (a) Mode 1, (b) Mode 2, (c) Mode 3 and (d) Mode 4 (continued) (see online version for colours)

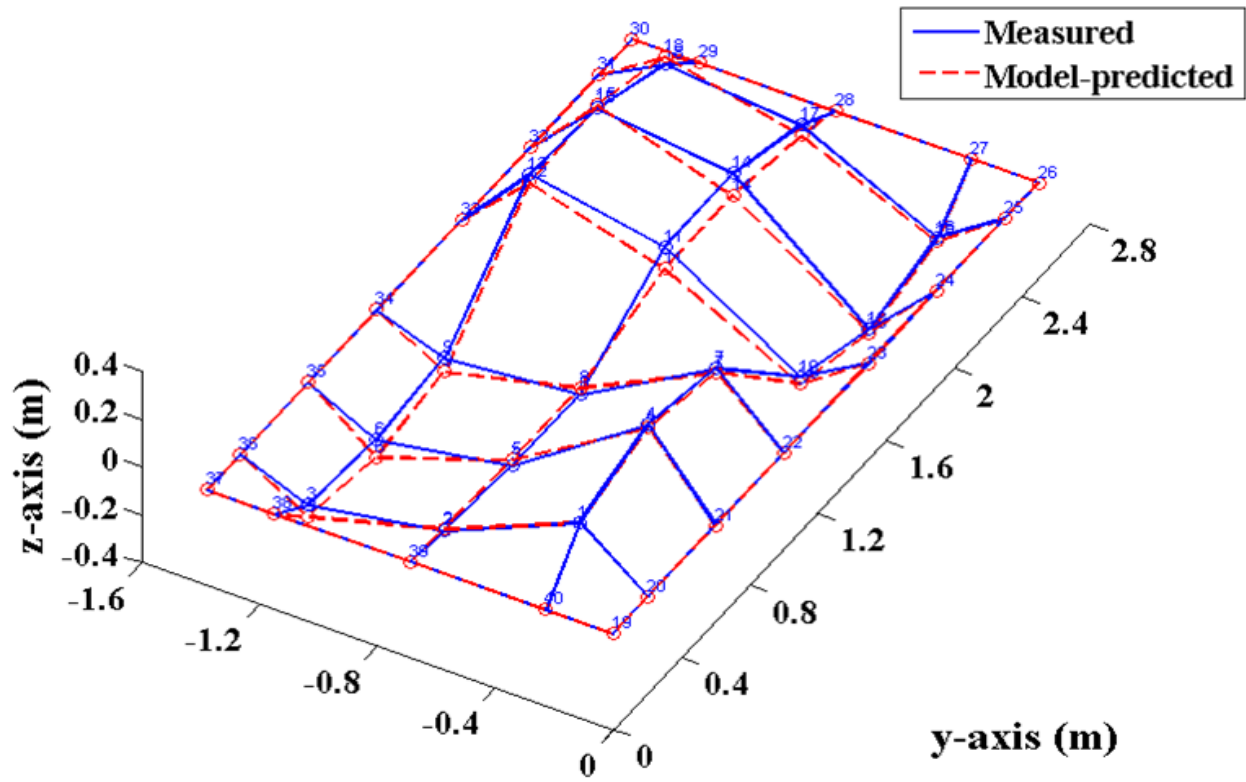

x-axis (m)

x-axis (m)

(c)

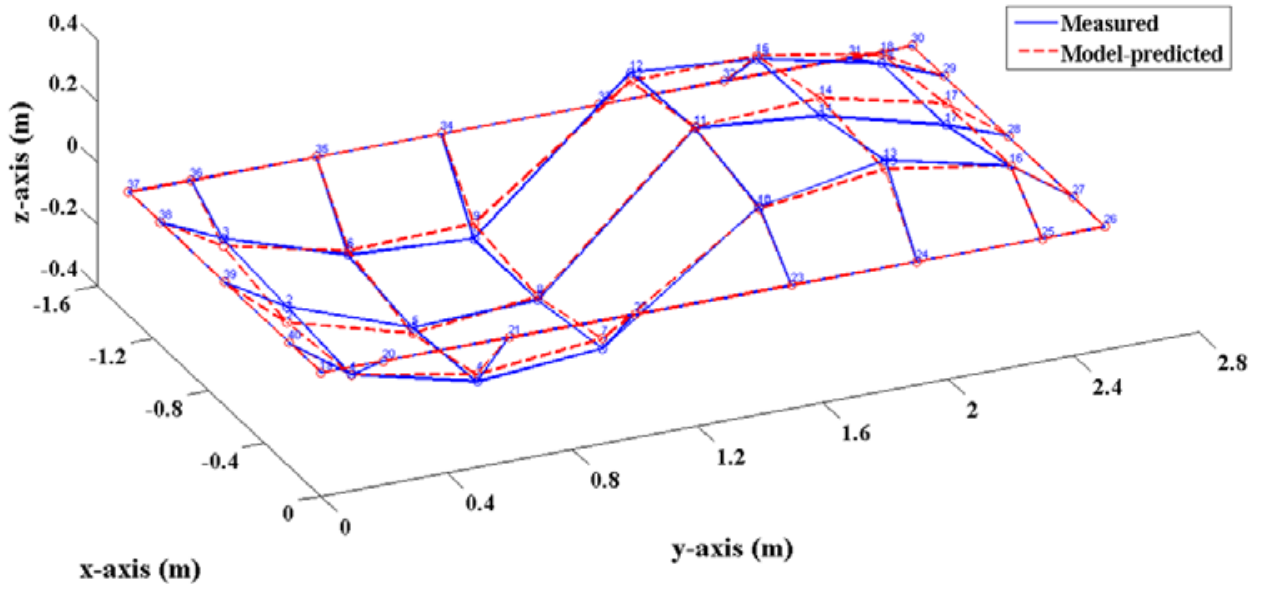

(d)

Table 2 MAC values between the measured and model-predicted mode shapes

\begin{tabular}{lc}
\hline Mode & $M A C$ \\
\hline Mode 1 & $93.71 \%$ \\
Mode 2 & $93.25 \%$ \\
Mode 3 & $89.93 \%$ \\
Mode 4 & $90.34 \%$ \\
\hline
\end{tabular}




\section{Conclusions}

This paper presents a comprehensive study of ambient vibration test, modal identification and model updating of a steel frame supported standing seam metal roofing model under laboratory condition. Modal parameters were extracted from the vibration data using FDD method and four vertical vibration modes were identified. To further quantify the stiffness of clips, a finite element model was developed in ANSYS and was updated following the MCMC-based Bayesian model updating method. The results show that an axial stiffness of $5.76 \times 10^{6} \mathrm{~N} / \mathrm{m}$ can be used for this kind of clips for dynamic analysis. The matching between measured and model-predicted modal parameters is in general acceptable after model updating. This study demonstrates the feasibility in identifying the clip stiffness of this kind of standing seam metal roofing system based on a set of measured vibration data. Based on the experience obtained from the indoor panel test, an in-situ field test will be carried out in the future to study the dynamic characteristics of metal roofs under operational condition. The findings presented in this paper are important for the development of a structural health monitoring system for metal roofing system in the future.

\section{Acknowledgements}

The work described in this paper was partially supported by a grant from the Research Grants Council of the Hong Kong Special Administrative Region, China [Project No. CityU 11210517 (GRF 9042509)]. The project leading to the publication of this paper is also partially funded by the Chinese National Engineering Research Centre for Steel Construction (Hong Kong Branch) (Project Nos. 1-BBY3 and 6) of the Hong Kong Polytechnic University. The authors would like to thank Mr. Pang Yiu Kwong, managing director, P\&Ls' Building Material (HK) Co., Ltd for the in-kind support of the metal roofing system together with the fabrication devices.

\section{References}

Au, S.K. and Beck, J.L. (1999) 'A new adaptive importance sampling scheme for reliability calculations', Structural Safety, Vol. 21, No. 2, pp.135-158.

Brincker, R., Zhang, L.M. and Andersen, P. (2001) 'Modal identification of output-only systems using frequency domain decomposition', Smart Materials and Structures, Vol. 10, No. 3, pp.441-445.

Damatty, A.A.E., Rahman, M. and Ragheb, O. (2003) 'Component testing and finite element modeling of standing seam roofs', Thin-Walled Structures, Vol. 41, No. 11, pp.1053-1072.

Farquhar, S., Kopp, G.A. and Surry, D. (2005) 'Wind tunnel and uniform pressure tests of a standing seam metal roof model', Journal of Structural Engineering, Vol. 131, No. 4, pp.650-659.

Feng, D. and Feng, M.Q. (2017) 'Identification of structural stiffness and excitation forces in time domain using noncontact vision-based displacement measurement', Journal of Sound and Vibration, Vol. 406, No. 21, pp.15-28.

Hastings, W.K. (1970) 'Monte Carlo sampling methods using Markov chains and their applications', Biometrika, Vol. 57, No. 1, pp.97-109. 
Hu, J. and Yang, J.H. (2018b) 'Operational modal analysis and Bayesian model updating of a coupled building', International Journal of Structural Stability and Dynamics, Vol. 19, No. 1, p.1940012.

Hu, J., Chui, T.H. and Lam, H.F. (2016) 'Vibration test and finite element modeling of a steel frame supported standing seam metal roof panel', Proceedings of the 8th International Conference on Steel and Aluminium Structures (ICSAS 2016), Hong Kong, China, 7-9 December, Paper No. 522, ISBN: 978-962-8014-26-2.

Hu, J., Lam, H.F. and Yang, J.H. (2018) 'Operational modal identification and finite element model updating of a coupled building following Bayesian approach', Structural Control and Health Monitoring, Vol. 25, No. 2, p.e2089.

Lam, H.F., Hu, J. and Yang, J. (2017a) 'Bayesian operational modal analysis and Markov chain Monte Carlo-based model updating of a factory building', Engineering Structures, Vol. 132, No. 3, pp.314-336.

Metropolis, N., Rosenbluth, A.W., Rosenbluth, M.N., Teller, A.H. and Teller, E. (1953) 'Equation of state calculations by fast computing machines', The Journal of Chemical Physics, Vol. 21, No. 6, pp.1087-1092.

Serrette, R. and Pekoz, T. (1995a) 'Distortional buckling of thin-walled beams/panels. I: theory', Journal of Structural Engineering, Vol. 121, No. 4, pp.757-766, ASCE.

Serrette, R. and Pekoz, T. (1995b) 'Distortional buckling of thin-walled beams/panels. II: design methods', Journal of Structural Engineering, Vol. 121, No. 4, pp.767-776, ASCE.

Sinno, R., Nail, J. and Fowler, S. (2001) Simulation of Nonuniform Unsteady Wind Pressures (Roof Uplift Test), MBMA 98-01 Report, July.

Yuen, K.V. and Katafygiotis, L.S. (2003) 'Bayesian fast Fourier transform approach for modal updating using ambient data', Advances in Structural Engineering, Vol. 6, No. 2, pp.81-95. 